\title{
Regularization of Diagrammatic Series with Zero Convergence Radius
}

\author{
Lode Pollet, ${ }^{1}$ Nikolay V. Prokof'ev, ${ }^{2,3}$ and Boris V. Svistunov ${ }^{2,3}$ \\ ${ }^{1}$ Department of Physics, Harvard University, Cambridge, Massachusetts 02138, USA \\ ${ }^{2}$ Department of Physics, University of Massachusetts, Amherst, MA 01003, USA \\ ${ }^{3}$ Russian Research Center "Kurchatov Institute", 123182 Moscow, Russia
}

(Dated: October 29, 2018)

\begin{abstract}
The divergence of perturbative expansions for vast majority of macroscopic systems, which follows from Dyson's collapse argument, prevents Feynman's diagrammatic technique from being directly used for controllable studies of strongly interacting systems. We show how the problem of divergence can be solved by replacing the original model with a convergent sequence of successive approximations which have a convergent perturbative series. As a prototypical model, we consider the zero-dimensional $|\psi|^{4}$ theory.

PACS numbers: 02.70.Ss, 05.10.Ln
\end{abstract}

Almost sixty years ago, Dyson provided a physical argument why power-series expansions in quantum electrodynamics are divergent even after proper charge and mass renormalization [1]. The argument is straightforwardly generalizable to a wide range of classical and quantum statistical problems and has far-reaching consequences for any perturbative approach [2]. It goes as follows: The interaction potential between two electrons with charge $e$ is of the form $e^{2} / r_{12}$, with $r_{12}$ the distance between them. When performing a series expansion in $e^{2}$ (i.e., the interaction strength) a physical quantity of interest can be expressed as

$$
F\left(e^{2}\right)=a_{0}+a_{2} e^{2}+a_{4} e^{4}+\ldots
$$

If the series have finite convergence radius $\xi$ then $F\left(e^{2}\right)$ is an analytic function at $e=0$, implying that for sufficiently small values of $e, F\left(-e^{2}\right)$ is a well-behaved analytic function. However, $F\left(-e^{2}\right)$ corresponds to a fictitious world with purely imaginary particle charges where the interaction potential is of the form $-e^{2} / r_{12}$, and is thus attractive. In this fictitious world, the vacuum state would be unstable against production of an infinite number of electron-positron pairs, each put in a separate region of space since the gain in the negative Coulomb energy is larger than the increase in the kinetic energy. Hence, Dyson concluded that $F\left(e^{2}\right)$ cannot be analytic around $e=0$ and that the convergence radius must be zero, $\xi=0$.

The $|\psi|^{4}$ theory is associated with the following partition function in $d$ dimensions:

$$
Z=\int \mathcal{D} \psi \mathrm{e}^{-\int d^{d} r\left\{|\nabla \psi|^{2}+\lambda|\psi|^{4}\right\}},
$$

describing the statistics of the classical complex-valued field $\psi(\mathbf{r})$. This effective field theory is often used to describe critical behavior of the superfluid phase transitions in interacting Bose systems. The model (2) can be solved numerically very efficiently by a number of methods, but none of them is based on an expansion in $\lambda$. A successful regularization of the perturbative expansion in $\lambda$ for the $|\psi|^{4}$ theory would change the status of the standard diagrammatic technique to that of a systematic method for accurate studies of strongly correlated systems, and provide the basis for numerical treatments in the framework of (bold) Diagrammatic Monte Carlo [3. Since Taylor expansion in $\lambda$ is subject to the Dyson's collapse argument, it is clear that some regularization of the theory is required by which we mean any sequence of field-theoretical approximations to the original model, controlled by regulator $N$, leading to a diagrammatic series with (at least) a finite convergence radius. The correct physical result is obtained after extrapolation of $N$ to infinity. As an example of such a regularization, we mention a continuousspace (finite-temperature) system of fermions with truncated single-particle momenta. Truncation sets the limit on the maximal density of the system, thereby saving the problem from Dyson's collapse and allowing one to employ the diagrammatic treatment up to high-order (see, e.g., [4). While we do not exclude the possibility that in specific cases the regularization procedure might ultimately be reduced to a re-summation scheme, we realize that a generic regularization protocol, in view of the mathematical ambiguity of restoring a function from an asymptotic series with zero convergence radius, should derive from the series provenance, i.e. explicitly depend on the form of the original theory. We stress that we seek a regularization which preserves the structure of diagrammatic expansions.

In this Letter, we introduce a class of regularization techniques that introduce counter-terms to the diagrammatic series. In this approach, the so-called "sign blessing", i.e. sign alternation of the same-order diagrams (cf. the fermionic case [5]) of a regularized series - as contrasted to the sign-definiteness of the same-order diagrams of the original theory - is responsible for mutual cancelation of contributions from the factorial number of diagrams of a given order and series convergence.

Focusing solely on the convergence properties, it is instructive 22 to reduce the theory 2 to its zero- 
dimensional extreme. In this case, the field $\psi(\mathbf{r})$ is replaced by a complex number $\psi$ and the partition function reduces to the integral $\left(x \equiv|\psi|^{2}\right)$

$$
I(\lambda)=\int_{0}^{\infty} d x \mathrm{e}^{-x-\lambda x^{2}}=\sqrt{\frac{\pi}{4 \lambda}} \mathrm{e}^{\frac{1}{4 \lambda}} \operatorname{erfc}\left(\frac{1}{2 \sqrt{\lambda}}\right) .
$$

In order to understand the convergence properties of the perturbative approximations in $\lambda$ for the $|\psi|^{4}$ theory, it suffices to do that for the integral $I(\lambda)$.

We start with identifying the problem for the standard Taylor series representation

$$
e^{-\lambda x^{2}}=\lim _{N \rightarrow \infty} \sum_{k=0}^{N} \frac{(-\lambda)^{k}}{k !} x^{2 k},
$$

which is that, reversing the order of taking the limit $N \rightarrow \infty$ and integrating over $x$, one obtains a series diverging as $\sim \lambda^{k}(2 k) ! / k !$. This is a hallmark for an asymptotic series with zero convergence radius. An equivalent observation is that for any finite $N$ the integral is dominated by values of $x$ where the finite sum is not providing an accurate description of the original exponent [6]. Using a different representation for the exponential function

$$
e^{-\lambda x^{2}}=\lim _{N \rightarrow \infty} Z_{N}(x)=\lim _{N \rightarrow \infty}\left(1-\frac{\lambda x^{2}}{N}\right)^{N},
$$

also fails for exactly the same reason. It formally produces a sequence of integrals $I_{N}(\lambda)$, by replacing $e^{-\lambda x^{2}} \rightarrow$ $Z_{N}(x)$, which takes us away from the original problem because the dominant contribution to $I_{N}(\lambda)$ comes from $x \sim N$, as contrasted to the scale $x \lesssim 1$ of the original integral. Equation (4) can be transformed into Eq. (5) by applying a resummation technique to the original Taylor series,

$$
\left(1-\frac{\lambda x^{2}}{N}\right)^{N} \equiv \sum_{k=0}^{N} \frac{(-\lambda)^{k}}{k !} x^{2 k} f(k, N),
$$

where the resummation function $f(k, N)=k ! C_{N}^{k} / N^{k}$ is such that $f(k \ll N) \approx 1$ and decreasing fast for $k \rightarrow N$. Formally, $f$ is supposed to suppress the leading divergence of the original series which is not the case for Eq. (5). One may seek other functions $f(k, N)$ rendering the extrapolation procedure $N \rightarrow \infty$ meaningful but as far as we know the solution was not found yet, nor is it clear that it exists. However, as we prove below, the solution does exist when the series are generalized to include additional counter-terms; namely, within the

$$
e^{-\lambda x^{2}}=\lim _{N \rightarrow \infty} \sum_{k=0} x^{k} f(k, N)
$$

representation, where $f(k, N)$ is restricting summation over $k$ to some polynomial of finite order which scales with $N$. We require that the expansion is in terms of integer powers of $x$ since otherwise the construction of Feynman's diagrams (which is our ultimate goal) becomes problematic. To generate the standard Feynman diagrammatic expansion based on Wick's theorem one has to introduce the exponential

$$
Z_{N}(x) \equiv \mathrm{e}^{\ln \left[Z_{N}(x)\right]},
$$

and expand the logarithm in powers of $x$.

Our solution is based on designing an alternative representation of the exponential function wich has to satisfy several requirements when expanded in powers of the coupling parameter. A successful perturbative approach has to satisfy the conditions of (i) convergence of the $\lambda$-expansion of $I_{N}(\lambda)$ for all $\lambda>0$ at any fixed $N$, and (ii) meaningful extrapolation to the $N \rightarrow \infty$ limit to ensure that $\lim _{N \rightarrow \infty} I_{N}(\lambda)=I(\lambda)$. Given that the theory (2) features non-analyticity at the second-order phase transition point, one might think that the condition (i) should be weakened to sufficiently small $\lambda$ 's. Nevertheless, we consciously require convergence everywhere to guarantee that the divergence of the $\lambda$-expansion of the field-theoretical counterpart of $I_{N}(\lambda)$ is exclusively due to the phase transition in the macroscopic limit; the convergence radius remaining infinite for any finite-size system (qualitatively analogous to our zero-dimensional model).

Let us introduce the parameterization

$$
y=u x^{2 / m}, \quad u=(\lambda / N)^{1 / m},
$$

with $m$ an integer number, and note that the most severe problem with Eq. (5) can be overcome by seeking a solution in the form

$$
\mathrm{e}^{-\lambda x^{2}}=\lim _{N \rightarrow \infty}[f(y)]^{N},
$$

with the function $f($.$) being bounded, 0 \leq f() \leq$.1 , along the real axis of $y$, and having an expansion

$$
f(y)=1-y^{m}+\ldots .
$$

The terms that are of a higher exponent than $m$ serve as counter terms compared to Eq. (5). These requirements guarantee that the series of integrals $I_{N}(\lambda)$ converge to the right answer, but this does not solve yet the problem with perturbative expansions unless we also require that the integral

$$
I_{N}(\lambda)=\int_{0}^{\infty} d x \mathrm{e}^{-x}[f(y(x))]^{N}
$$

is convergent for any complex value of $u$ [see Eq. (9)], which is the necessary condition for $I_{N}(\lambda)$ to be expandable in the convergent Taylor series in powers of $u$. It is this condition which forces one to consider $m>1$.

It turns out that there are infinitely many functions corresponding to $m=4$ ( $m=4$ is in practice the obvious 
choice), all satisfying the above-formulated requirements. Here we present one of them which is based on Bessel functions $J_{n}$ :

$$
f(y)=J_{0}(2 z)+2 J_{2}(2 z)+\frac{5}{3} J_{4}(2 z)
$$

where $z=(72)^{1 / 4} y$ and the explicit values of coefficients follow from the requirement (11). With regards to all conditions we find:

- For any positive $u$ the condition $0 \leq f() \leq$.1 means that all integrals 12 are dominated by the $x \lesssim 1$ region and thus guaranteeing that the condition (ii) is satisfied. - $[f(y)]^{N}$ is an entire function with infinite convergence radius.

- The integrals 12 converge for any complex $u$ since the strongest divergence at $x \rightarrow \infty$ (occurring at imaginary $u)$ is exponential with the exponent $\propto \sqrt{x}$ and thus not dangerous in view of the $e^{-x}$ factor; the condition (i) is thus satisfied.

- the Taylor expansion of $f(y)$ is in even powers of the argument. Hence, the requirement of having only integer powers of $x$ is also satisfied since $y^{2}=u^{2} x$.

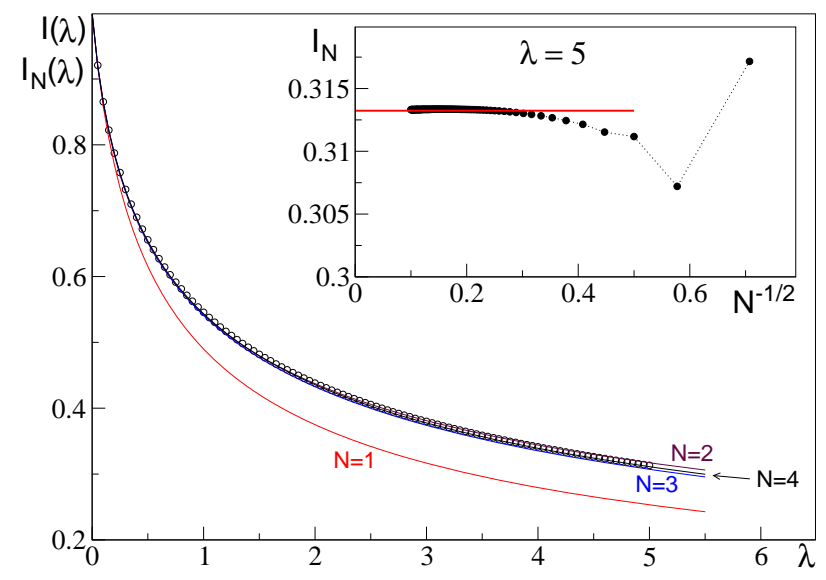

FIG. 1: (color online) Convergence properties of the sequence $I_{N}(\lambda)$ with $f(y)$ defined in Eq. [13). The result for $I(\lambda)$ is shown with circles. The convergence with $N$ for $\lambda=5$ is shown in the inset.

The convergence with $N$ of the sequence of integrals 12 is illustrated in Fig. 1. Remarkably, $N=4$ already produces results which are accurate at the $1 \%$ level up to $\lambda=5$. The ultimate convergence to the exact solution is a polynomial function of $N^{-1 / 2}$ and can be easily extrapolated from finite $N$. A peculiar feature of the arising theory is that perturbative expansion is in terms of $u$ which is a fractional power of $\lambda$.

In order to comply with Feynman's diagrammatic rules we need to study integrals over $x$ for Taylor expansions

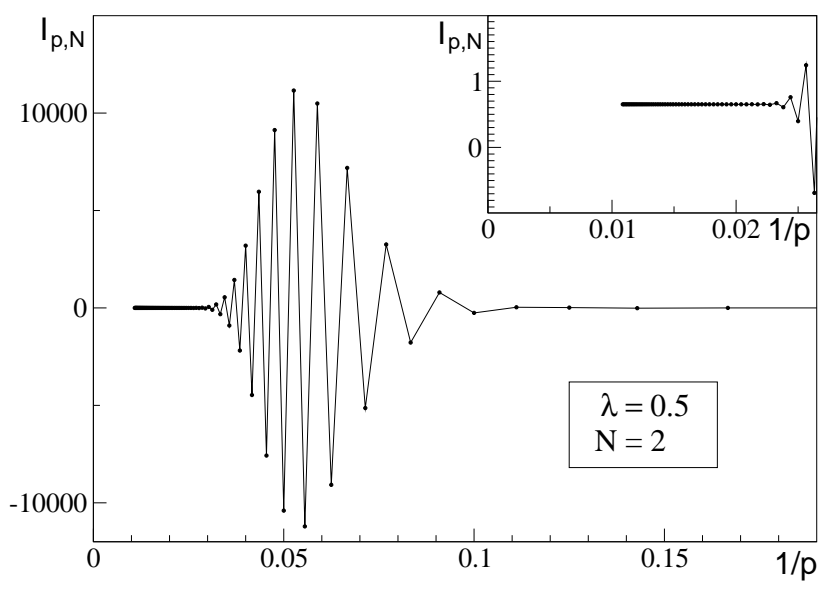

FIG. 2: Plotted are integrals $I_{p, N}=\int d x e^{-x} Z_{p, N}(x)$ where $Z_{p, N}$ is the Taylor expansion of $[f(y)]^{N}$ up to order $x^{p}$, for $\lambda=0.5$. In this example $N=2$ which guarantees that the converged answer is accurate down to one percent accuracy.

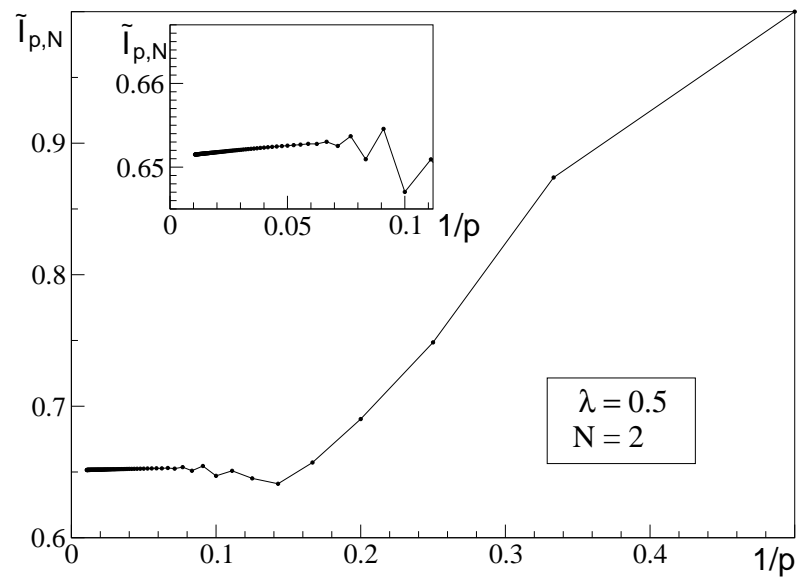

FIG. 3: Integrals $\tilde{I}_{p, N}=\int d x e^{-x} \tilde{Z}_{p, N}(x)$ based on the same series as in Fig. 2 resummed using the near-Gaussian function, see text. The elimination of the severe sign-problem and the radical improvement of convergence properties suggests that polynomial size and optimization should be explored.

$Z_{N}(x)=\sum_{k=0}^{\infty} x^{k} C_{k}$ done up to order $p$. The implications for that are shown in Fig. 2 where we plot the final result when $Z_{N}(x)$ is replaced with $Z_{p, N}(x)=$ $\sum_{k=0}^{p} x^{k} C_{k}$. At first, the impression is that a severe signproblem and a very high-order expansion is the price for obtaining a set of approximations using series with infinite convergence radius. When we take stronger $\lambda$ or larger $N$, the fluctuations that need to cancel against each other become larger, and the minimum Taylor ex- 
pansion order increases. However, when the original series are further modified using resummation technique based on the near-Gaussian function

$$
Z_{p, N}(x) \rightarrow \tilde{Z}_{p, N}(x)=\sum_{k=0}^{p} x^{k} C_{k} e^{-k^{s} / p}, \quad s=2.05,
$$

we obtain results shown in Fig. 3 Amazingly, the sign problem between the expansion orders is essentially eliminated and accurate results can be obtained already for relatively small expansion orders $\sim 5$, making the entire scheme a viable solution to the regularization of diagrammatic expansions with zero-convergence radius (increasing $N$ does not change the picture substantially).

At this point we note that series convergence for fixed $N$ allows us to truncate the series at some order $p_{*}(N)$ such that the difference between $I_{p_{*}, N}=$ $\int d x e^{-x} Z_{p_{*}, N}(x)$ and $I_{N}$ is smaller than $\left|I_{N}-I(\lambda)\right|$. This would account for the regularization technique in the form (7) when the set of approximations to the exponential function is always in the form of a finite-order polynomial in $x$. Clearly, there is a lot of room for optimizing the form of such polynomials, and the success of the near-Gaussian resummation, which in essence performs such an optimization, proves the point-radical improvements in efficiency are possible along these lines even with relatively small $p_{*}$. One of such approaches (tested for our model with quite satisfactory results) is based on polynomials that reproduce exactly the first $p_{*}$ moments of the distribution $e^{-x-\lambda x^{2}}$.

To deal with a generic non-local coupling between fields $e^{-\lambda\left|\psi_{1}\right|^{2}\left|\psi_{2}\right|^{2}} \equiv e^{-\lambda x_{1} x_{2}}$ we propose to start with the following regularization (later on it can be further modified/optimized)

$$
e^{-\lambda x_{1} x_{2}}=\lim _{N \rightarrow \infty}\left[g\left(y_{1}\right) g\left(y_{2}\right)-\frac{\lambda x_{1} x_{2}}{N} f\left(y_{1}\right) f\left(y_{2}\right)\right]^{N},
$$

where $y_{i}=u^{\prime} x_{i}^{1 / 2}$, and $u^{\prime}=\left(1 / N^{a}\right)^{1 / 2}$ with the exponent $a<1 / 2$. The entire functions $g(y)$ and $f(y)$ have to satisfy criteria similar to those mentioned above: be even functions of $y$, decay on the real axis, and diverge not faster then $e^{|y|}$ in the complex plane. These requirements are satisfied if $f$ and $g$ are based on linear combinations of Bessel functions. We also demand that $f(0)=g(0)=1$, the Taylor series expansion of $g$ starts from an order $y^{2 s}$ with as $>1$, and the modulus of expression in parentheses is smaller than unity. This also constitutes no problem using proper linear superpositions of $J_{n}$. The resulting regularization is guaranteed to work and, most importantly, it can be generalized to deal with arbitrary interaction potentials (two-body, three-body, etc.) because the $f$ and $g$ functions are defined in terms of local fields only. We had to proceed in this way instead of repeating the solution of the single-field problem because the expansion in $y^{2} \propto\left|\psi_{1}\right|\left|\psi_{2}\right|$ does not allow the diagrammatic technique, contrary to $y_{i}^{2} \propto\left|\psi_{i}\right|^{2}$.
The other important difference between Eqs. 15 and $(10)$ is that the $y$ variables in Eq. (10) do not contain the parameter $\lambda$, and the diagrammatic expansion goes thus in integer powers of $\lambda$ and $u^{\prime 2}$. We believe that with an appropriate choice of functions it is possible to proceed with $a=1 / 2$ and $u^{\prime}=(\lambda / N)^{1 / 4}$, just as before. This approach has the "advantage" of being based on a single parameter expansion. Likewise, one may apply (15) with $a<1 / 2$ and an $\lambda$-independent parameter $u^{\prime}$ to deal with the single-site regularization.

In conclusion, diagrammatic series with zero convergence radius can be dealt with by approximating the original interaction exponential with an order- $p$ polynomial in the integer powers of the original interaction and regularizing counter-terms. The action of the regularized theory is obtained by exponentiating the polynomial. The accuracy of the approximation is controlled by the parameter $p$. While the polynomial has to satisfy a number of requirements guaranteeing, in particular, that the sequence of approximate theories approaches the original one at $p \rightarrow \infty$ (counterintuitively, this property requires special care), there is still a continuum of possible choices, with some characteristic examples tested on the basis of zero-dimensional $|\psi|^{4}$ model. We believe that the outlined approach - especially in the context of Diagrammatic Monte Carlo - opens up an opportunity to utilize Feynman's diagrams as a generic tool to address strongly correlated classical- and quantum-field systems.

We acknowledge valuable and stimulating discussions with M. Ogilvie and Y. Meurice during our stay at the Aspen Center for Physics. This work was supported by the Swiss National Science foundation, the National Science Foundation grant PHY-0653183, and by a grant from the Army Research Office with funding from the DARPA OLE program.

[1] F. J. Dyson, Phys. Rev. 85, 631 (1952).

[2] J. W. Negele and H. Orland, Quantum Many-Particle Systems, Westview Press, Boulder, 1998.

[3] N. V. Prokof'ev and B. V. Svistunov, Phys. Rev. Lett. 99, 250201 (2007).

[4] E. Burovski, E. Kozik, N. Prokof'ev, B. Svistunov, and M. Troyer, Phys. Rev. Lett. 101, 090402 (2008).

[5] N. Prokof'ev and B. Svistunov, Phys. Rev. B 77, 020408 (2008); K. Van Houcke, E. Kozik, N. Prokof'ev, and B. Svistunov, Diagrammatic Monte Carlo, [in Computer Simulation Studies in Condensed Matter Physics XXI, Eds. D.P. Landau, S.P. Lewis, and H.B. Schuttler (Springer Verlag, Heidelberg, Berlin 2008)]; E. Kozik, K. Van Houcke, E. Gull, L. Pollet, N. Prokof'ev, B. Svistunov, and M. Troyer, EPL 90, 10004 (2010).

[6] The idea of our approach to series regularization was inspired by Y. Meurice's discussion of how naive regularization methods fail. 